\title{
A Variational Solution of the Schrödinger Equation in an Inhomogeneous Central Field
}

\author{
Ilya Tolstov ${ }^{1,3, \star}$, Boris Freinkman ${ }^{2, \star \star}$, and Sergey Polyakov ${ }^{3, \star \star \star}$ \\ ${ }^{1}$ Keldysh Institute of Applied Mathematics Miusskaya sq., 4 Moscow, 125047, Russia
}

\begin{abstract}
The present work is devoted to the computer modeling of emission processes from the graphene surface which is a promising material for modern applications. We examine the effect of the ion field inhomogeneity on the variational solution of the Schrödinger equation for the ground state of a loosely bound electron of a hydrogenlike carbon atom, which simulates the graphene model. This shows a significant impact of the allowance for the field inhomogeneity to the ground state of the electron.
\end{abstract}

\section{Introduction}

Currently, there is a huge amount of publications about field emission properties of various carbon nanostructures. Almost in all papers, the authors note the discrepancy between the experimental data obtained on the geometric form and parameters of field emission [1]. One of the important problems of the use of graphene and nanotubes on its basis in the nanotechnology is the lack of reliable data on the quantum states of atoms of graphene lattice in the presence of external field sources. Thus, for promising and potentially possible appliances of nanotubes as a cantilever in tunneling microscopes or as an electron source [2] in probe electron-optical systems, mathematical modeling is needed to determine their emission characteristics. For this, it is necessary to develop a model describing the interaction of the electron with the surface. In the previous work [3], the graphene model in the form of the lattice of hydrogen-like atoms with a screened ion in the Brandt model was used to calculate the ground state of atoms of the graphene surface [4]. Also, for calculation the ground state of an electron we used a variational solution of the Schrodinger equation, based on minimizing the electron potential energy in the ion field, that assumes homogeneity of the ion field. However, the field of the shielded and the singly ionized carbon atom in the Brant model was not homogeneous.

Within the hydrogen-like atom model used to describe the carbon atom in the graphene lattice the problem is regarded as the electron interaction with the ion lattice having some effective charge $q$. The Brandt-Kitagawa model [4] is an appropriate a model describing the inhomogeheous interaction.

\footnotetext{
$\star$ e-mail: tolstov_ilya@inbox.ru

$\star \star$ e-mail: bgfreink@gmail.com

$\star \star \star$ e-mail: polyakov@imamod.ru
} 


\section{Allowance for the inhomogeneity of the field for the hydrogen-like atom model}

In the previous work [3] the ground state of an electron in the singly ionized hydrogen-carbon atom was determined by finding an effective homogeneous field $\tilde{U}=-\frac{q}{r}+A$ from the condition of residual error minimum of the electron potential energy in the s-state in this field and in the ion field in the Brandt model [4]

$$
U(r)=\frac{1}{r}+\frac{Z-1}{r} \exp \left(-\frac{r}{\lambda}\right), \quad \lambda=g \frac{N_{e} / Z^{2 / 3}}{1-N_{e} / 7 Z} \frac{a_{0}}{Z^{1 / 3}},
$$

where $g=0.48, Z=6, N=5, \lambda=0.2655$.

The effective charge of the ion q was determined from the numerical solution of the equation:

$$
J(q)=\frac{q^{2}}{2 n^{2}}+\int_{0}^{\infty} R_{n s}^{2}(r, q) V(r) r^{2} d r
$$

However, the ion field in the Brandt model is not homogeneous along the radius since

$$
\frac{\partial}{\partial r} U_{i}(r)=-\frac{U_{i}(r)}{r}-\frac{Z-1}{\lambda_{i}} \neq \chi \frac{U_{i}(r)}{r} .
$$

According to [5], the total energy of a finite motion in an inhomogeneous central field is

$$
E=\frac{r}{2} \frac{\partial U}{\partial r}+U
$$

therefore, the effective charge $\mathrm{q}$ of an equivalent homogeneous field for the s-status should be determined from the condition of residual error minimum of an electron total energy in the s-state in this field and in the ion field in the Brandt model.

In this case, the effective charge $q$ will be determined from the solution of the variational equation

$$
J_{2}(q)=\left\langle R_{s}^{*}(x)\left[\frac{r}{2} \frac{\partial}{\partial r} U_{i}(r)+U_{i}(r)\right] R_{s}(x)\right\rangle-\frac{q^{2}}{2 n^{2}}, \quad x=\frac{2 q}{n} r .
$$

\section{Results}

A numerical results are proposed on figures 1-3. Figure 1 shows the distribution of the values calculated in this paper. Figure 2 shows the numerical solution of (2) and (5). Figure 3 shows the distribution of the effective homogeneous field in these two solutions.

As can be seen from the graphs, the results are significantly different. The calculated values of the effective charge are given in Table 1.

Table 1. Calculated values for charge and minimal energy

\begin{tabular}{lll}
\hline & minimal potential energy & minimal total energy \\
\hline $\mathrm{Q}$ & 0.71218754 & 0.4473579 \\
$\mathrm{~A}$ & 0.0836779 & 0.02791427 \\
$\mathrm{E}$ & 2.4214 & 1.5208 \\
\hline
\end{tabular}

The variation of the values computed for the lowest energy within the two formulations of the problem reaches $37 \%$. 

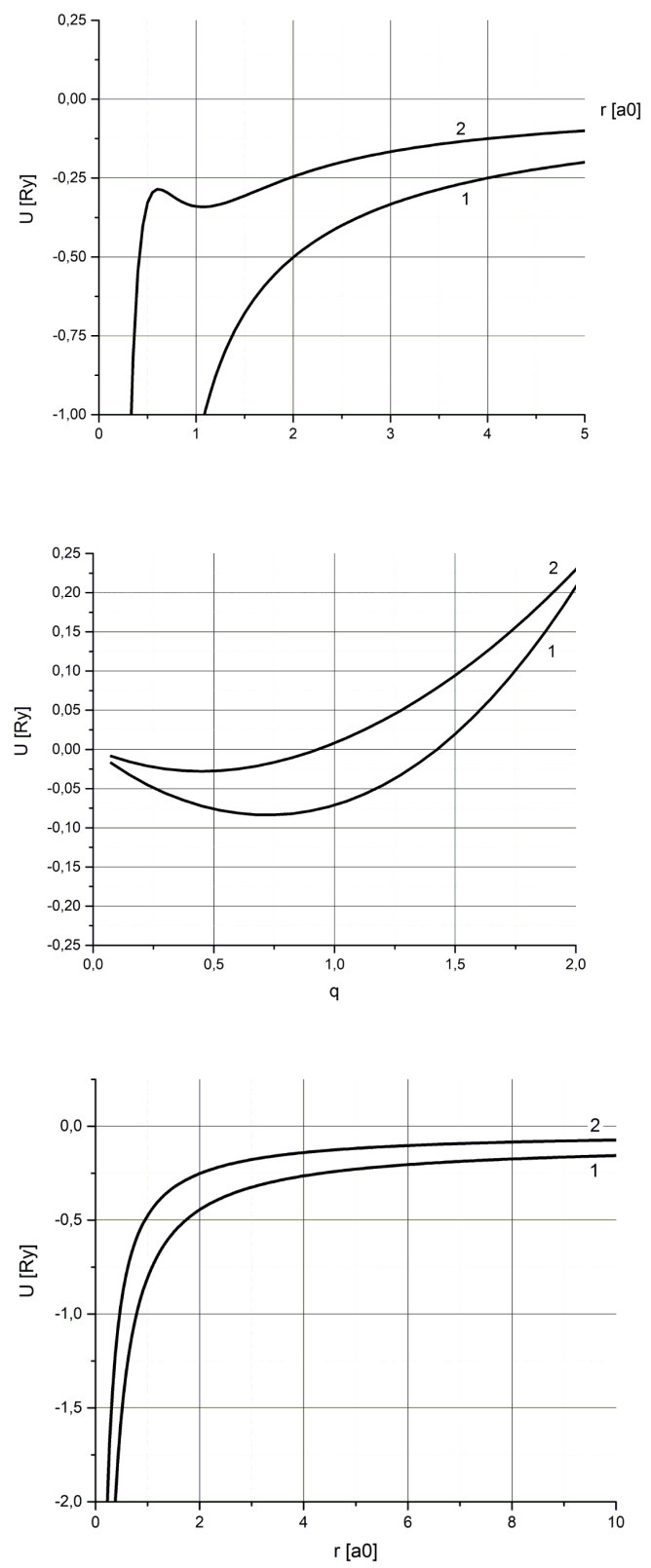

Figure 1. The distribution of the potential created by the effective charge of the ion (line 1) and the mean total energy (line 2). Here and below, the potential is measured in Rydberg.

Figure 2. Solution of the variational problem for a homogeneous (line 1) and inhomogeneous (line 2) case

Figure 3. Distribution of a homogeneous field of a hydrogen-like atom without taking into account (line 1) and taking into account (line 2) the virial theorem

\section{Conclusion}

These results indicate that during calculation of the ground state of an electron of the hydrogen-like atom with a shielded ion in the Brandt model, we should take into account the inhomogeneity of the ion field in the Brandt model.

The model under development will allow to predict the emission and transport characteristics of the devices based on graphene, which is necessary for the further progress of the electron microscopy. 
The main advantage of the model under development is its flexibility: the Brandt-Kitagawa potential allows to describe atomic charge and different degrees of ionization. Therefore, it is possible to calculate the surface potential for silicon.

\section{Acknowledgments}

This work was supported by the Russian Foundation for Basic Researches (project No. 17-01-00973a).

\section{References}

[1] K. Asano, Journal of Electrostatics, 68, 132-137 (2010)

[2] A.V. Eletskii, Physics Uspekhi, 53, 863-892 (2010)

[3] B.G. Freinkman, Matematicheskoe modelirovanie 27, 122-128 (2015) (in russ.)

[4] W. Brandt, M. Kitagawa, Phys. Rev. B 25, 5631-5637 (1982)

[5] A.C. Phillips, Introduction to Quantum Mechanics (Wiley, New York, 2003) 